\title{
Be there on time: Spatial-temporal regularities guide young children's attention in dynamic environments
}

\author{
Nir Shalev ${ }^{1,2 \star}$, Sage Boettcher ${ }^{1,2 \star}$, Hannah Wilkinson ${ }^{1}$, Gaia Scerif ${ }^{1}$, Kia Nobre ${ }^{1,2}$ \\ * authors contributed equally \\ 'Department of Experimental Psychology, University of Oxford, Oxford, UK \\ ${ }^{2}$ Oxford Centre for Human Brain Activity, Wellcome Centre for Integrative Neuroimaging, \\ Department of Psychiatry, University of Oxford, Oxford, UK
}

Corresponding author:

Nir Shalev

University of Oxford

Department of Experimental Psychology

Brain \& Cognition Lab

Oxford Centre for Human Brain Activity

Mail: nir.shalev@psy.ox.ac.uk

Keywords:

Visual search; Cognitive Development; Attention

Acknowledgments:

This research was funded by a Wellcome Trust Senior Investigator Award (104571/Z/14/Z) and a James S. McDonnell Foundation Understanding Human Cognition Collaborative Award (220020448) to A.C.N., and by the NIHR Oxford Health Biomedical Research Centre. The Wellcome Centre for Integrative Neuroimaging is supported by core funding from the Wellcome Trust (203139/Z/16/Z). 


\begin{abstract}
It is believed that children have difficulties in guiding attention while facing distraction. However, developmental accounts of spatial attention rely on traditional search designs using static displays. In real life, dynamic environments can embed regularities that afford anticipation and benefit performance. We developed a dynamic visual-search task to test the ability of children to benefit from spatiotemporal regularities to detect goal-relevant targets appearing within an extended dynamic context amidst irrelevant distracting stimuli. We compared children and adults in detecting predictable vs. unpredictable targets fading in and out among competing distracting stimuli. While overall search performance was poorer in children, both groups detected more predictable targets. This effect was confined to task-relevant information. Additionally, we report how predictions are related to individual differences in attention. Altogether, our results indicate a striking capacity of prediction-led guidance towards task-relevant information in dynamic environments, refining traditional views about poor goal-driven attention in childhood.
\end{abstract}




\section{Significance statement}

We present unique evidence for goal-driven guidance of spatial attention in young children. Using a new dynamic-visual-search task, we show that five-year-olds are comparable to adults in their ability to use prediction to guide spatial attention in a dynamic visual-search task. We dissociate prediction-led behaviour, which remained invariant across age groups, from search performance which varied. Prediction-led behaviour was confined to task-relevant information, providing further evidence for goal-driven behaviour across ages. Finally, we report an association between task markers and individual differences in attention. Altogether, we propose a refined perspective of current views on attention control in young children. The new experimental approach provides a platform for systematically investigating how goals, distractions, predictions, and their interactions guide behaviour across development. 


\section{Introduction}

Many tasks in real-life situations require us to manage multiple dynamic sources of information successfully. However, even for neurotypical adults, it is impossible to process all concurrent stimuli in complex and dynamically changing environments in their entirety. Instead, to behave adaptively, it is necessary to prioritise and select task-relevant information according to our goals and to reject irrelevant, distracting information. For example, crossing a busy road requires paying attention to a changing and hazardous environment while selectively prioritising critical signals at different locations and timings (traffic lights, car signals, etc.) and ignoring equally salient signals (advertisement boards, flying birds, etc.).

Avoiding hazards can be particularly challenging for children. Thus, we would be alarmed if a 5-year-old were to cross a busy street on her own, given children's proposed reduced capacity to ignore distraction and to focus on relevant information efficiently (Amso \& Scerif, 2015; Colombo, 2001; Johnson, 2001; Scerif, 2010). Salient events tend to capture children's attention (e.g., a noisy motorbike) and divert them from goal-relevant streams of information (e.g., the traffic on the road) (Gaspelin, Margett-Jordan, \& Ruthruff, 2015). The presence of multiple competing perceptual events is likely to exacerbate the problem (Kim \& Kastner, 2019).

When studying selection, competition, and distraction, visual search tasks are a popular choice (Wolfe, 2020). In a typical visual search task, observers search for a target appearing among a static display of distractors. It is widely recognised among developmental researchers that children perform poorly in visual search tasks compared to adults (Donnelly et al., 2007; Trick \& Enns, 1998). Proficiency in searching for targets among distractors with different combinations of the same features develops gradually into adolescence, with a marked improvement at around 6-7 years (Hommel, Li, \& Li, 2004; Lobaugh, Cole, \& Rovet, 1998; Merrill \& Lookadoo, 2004; Olafsdóttir, Kristjánsson, Gestsdóttir, Jóhannesson, \& Kristjánsson, 2016; Whitebread \& Neilson, 2000). These age-related differences are often attributed to the interaction between immature top-down attention-control mechanisms and developing sensory processes (Donnelly et al., 2007; Kim \& Kastner, 2019).

Therefore, studies of visual search in children highlight a core limitation of the developing mind in controlling attention endogenously. However, interestingly, children have been proposed to display a relative strength in absorbing new information, compared to adults. This complementary ability has been proposed to result from an inherent trade-off between top-down cognitive control vs. exploratory cognitive flexibility (Gopnik et al., 2017). According to this view, gaining life experience alters the way we interact with the environment. Improvements in 
cognitive control into adulthood are coupled with a tendency to be less exploratory and, as such, to rely more heavily on prior experience to exploit and maximise utility in a given context. In contrast, during childhood, exploratory behaviour may be more adaptive, serving to amplify learning about the environment, which may take precedence and help shape subsequent knowledge structures that come to guide behaviour in the future. Consequently, children outperform adults in tasks requiring the extraction of unusual causal patterns, for example in learning how to operate a machine based on counterintuitive rules (Lucas, Bridgers, Griffiths, \& Gopnik, 2014), and are also more likely to encode task-irrelevant information (Plebanek \& Sloutsky, 2017; Sloutsky \& Fisher, 2004).

Poor visual search performance in childhood sits well with the notion of poor goaldirected behaviour and elevated exploration during structured but challenging tasks that benefit from maximum exploitation. However, when deploying attention in novel and dynamic environments, learning and exploration may be equally beneficial. Ultimately, in order to understand how the different qualities of children's cognition guide adaptive behaviour it is necessary to upgrade visual search tasks to capture the ecological aspects of real-life situations. In extended dynamic contexts, the anticipation of events based on previous experience plays a fundamental role in guiding attention proactively to the right place at the right time. In a series of experiments, we recently demonstrated that when performing a visual-search task that extends over time, young adults can implicitly learn spatial and temporal regularities about the appearance of task-relevant items and then use these predictable patterns to improve performance (Shalev, Boettcher, \& Nobre, 2019). Our experimental framework differs from typical studies using informative cues to study event anticipation; instead, we embed spatial and temporal predictions implicitly within multiple dynamic, competing and temporally extended events.

Interestingly, in contrast to visual-search studies, in which children (and even adolescents) often perform poorly when compared with adults (e.g., Donnelly et al., 2007; Trick \& Enns, 1998), cueing tasks reveal that children have adult-like capacity to orient attention in space (e.g., Johnson, Posner, \& Rothbart, 1991) and time (e.g., Droit-Volet \& Coull, 2016). Indeed, the ability to anticipate a stimulus based on implicit temporal regularities has been noted as early as infancy, as babies react to the omission of a regular, predictable stimulus (e.g., Brackbill \& Fitzgerald, 1972; Colombo \& Richman, 2002; Mento \& Valenza, 2016). In older age groups, four- and five-year old children can extract task-embedded temporal properties to anticipate upcoming events (e.g., Droit-Volet \& Coull, 2016; Johnson, Burrowes, \& Coull, 2015; Mento \& Granziol, 2020; Vallesi \& Shallice, 2007). These studies indicate that young 
children can control attention in time. However, the tasks used so far are restricted to testing responses to single targets occurring alone within temporally confined trials, and do not yet address the ability of using temporal orienting to select targets among competing signals in dynamic and temporally extended contexts.

Additional evidence for children's ability to control temporal attention comes from studies of statistical learning, in which they exhibit the capacity of encoding highly complex structures that extend beyond discrete cue-interval associations (see Saffran \& Kirkham, 2018; Thiessen, 2017). However, the statistical learning literature in young children is normally focused on auditory and linguistic signals (e.g., Estes, Evans, Alibali, \& Saffran, 2007; Saffran, 2001). Visual studies have examined probabilistic learning of spatial (e.g., Bertels, San Anton, Gebuis, \& Destrebecqz, 2017; Tummeltshammer, Amso, French, \& Kirkham, 2017), or sequential (e.g., Fiser \& Aslin, 2002; Kirkham, Slemmer, \& Johnson, 2002; Wu, Gopnik, Richardson, \& Kirkham, 2011) relations between successive items in a stream. In contrast, here we target children's use of spatio-temporally extended regularities in complex and competitive environments.

In unfolding real-life tasks, neither spatial nor temporal structures are held constant (as in laboratory search or cueing tasks), and prior experience of environmental regularities contributes to all aspects of guidance. Whereas previous studies on spatial cueing, control of temporal attention, and statistical learning provide a promising foundation, it remains unclear whether children can extract and utilise concurrent spatial and temporal predictions about the occurrence of relevant events occurring within dynamic contexts and amongst competing task-irrelevant distractors. Traditional views, rooted in developmental studies of visual search, emphasise the reduced capacity for goal-directed attentional guidance early in development. This view would be critically challenged if children were shown to utilise spatio-temporal structure in a goal-driven manner when facing competing sensory inputs.

In the current study, we build on the foundations of static visual search, temporal orienting, and statistical-learning tasks to develop an experimental framework for investigating whether children can use anticipation to guide attention in complex and dynamically changing contexts. This is akin to natural environments changing in space and time - like a busy street. We aim to learn whether spatial-temporal regularities can benefit young observers performing a dynamic visual search. In our task, regularities are embedded within a succession of visual stimuli occurring over extended trials, in principle allowing observers to predict when and where some of the targets will appear but not others. We hypothesised that, while children may find 
fewer targets overall compared to adults, they may nevertheless learn temporal and spatial regularities to guide attention in a goal-driven way. In addition to looking at age-related differences between young children (5-6 years old) and adults, we also tested whether the capacity to form predictions was associated with children's age or with behavioural markers of typical attention development.

\section{Methods}

All experimental procedures and protocols were reviewed and approved by the University of Oxford Central University Research Ethics Committee.

We adapted the Dynamic Visual Search (DVS) task we recently developed to investigate whether children and adults use task-embedded spatial and temporal regularities to guide selective attention within extended contexts. The experimental approach was developed and validated in a series of experiments testing more than a hundred young adults (Shalev, Boettcher, et al., 2019).

Participants performed a visual search in a dynamic display in which all stimuli faded in and out over extended trials. On every trial, they were requested to indicate where they detected the pre-designated target stimuli (e.g., aeroplanes) among a competing dynamic array of distracting stimuli (e.g., butterflies). The central withinparticipant experimental manipulation involved the spatiotemporal predictability of a subset of target stimuli: Half of the targets consistently appeared at predictable quadrants and onsets, whereas the other half were unpredictable. The experimental task allowed us to compare performance (i.e., hits and false alarms) in different age groups (children vs. adults) as well as their ability to use spatiotemporal predictions to benefit performance. To make the task more engaging, pictures of an aeroplane, dragonfly, and birds acted as targets and distractors. The identical task and parameters were used to test both children and adults.

\section{Participants}

Adults: The adult sample consisted of 25 undergraduate students ( 20 female) with mean age 20.08 years $(S D=1.66 \mathrm{yr}$ ). We recruited healthy adults with normal or corrected-to-normal vision through advertising on student Facebook pages and college noticeboards. They gave informed consent and were compensated for their time (rate of $£ 10$ per hour).

Children: The child sample consisted of 80 children (41 male, 39 female) with mean age $5.58 \mathrm{yr}$. (mean age in months was $67 ; S D=4.03 \mathrm{mo}$.). After a primary state school 
agreed to participate, information letters with opt-out forms were sent to parents of children at their first year of primary education ('Year 1'). Children were included unless parents submitted opt-out forms.

\section{Cognitive Task: Dynamic Visual Search}

Apparatus. Children were tested in a dedicated room at their school. Young adults were tested in a testing booth within the Department of Experimental Psychology, University of Oxford. In both cases, participants sat in a quiet, dimly-lit room, near a desk with the tablet on it. The tablet, a $10^{\prime \prime}$ Microsoft Surface tablet $(28 \times 17 \mathrm{~cm} ; 60 \mathrm{~Hz}$ refresh rate) running Linux, was placed within comfortable reach of the participant's hand. The tablet was standing at an upright position at $80^{\circ}$ to the table by folding its cover. The experimental script was generated using Psychophysics Toolbox (Brainard, 1997) in MATLAB (version 2014b, The Mathworks Inc., Natick, NA, USA).

Stimuli. Figure 1 shows the task. Participants were instructed to find eight targets on each trial. Trials lasted approximately fourteen seconds, and consisted of eight targets and sixteen distracting stimuli fading in and out of the search display independently over its duration. The search display consisted of four unique $1 / \mathrm{F}$ static noise patches that were generated for each trial. The four quadrants of the display were clearly separated, and each extended approximately $11^{\circ}$ (horizontal angle) $\times 7^{\circ}$ (vertical angle) $(98 \mathrm{~mm} \times 61 \mathrm{~mm})$. Three different object stimuli were used as targets and distractors: an aeroplane, a dragonfly, and a bird. All stimuli were black with transparent backgrounds.

At the beginning of the experiment, each participant was randomly assigned a target (e.g., an aeroplane) and was told to ignore distractors (e.g., dragonfly and bird). Stimuli faded slowly in and out of view, but did not move. Fade-in time was set to 1.3 seconds (gradually becoming visible over 80 refresh-rate cycles until reaching maximum visibility). The target stayed on the screen for another 1.3 seconds, and then faded out over 1.3 seconds. Each stimulus was about $2 \mathrm{~cm}$ in length and width, which roughly translates to $\sim 2.5^{\circ}$ (assuming a mean distance of $45 \mathrm{~cm}$ ) and could appear anywhere in one of the four quadrants as long as it did not overlap with another stimulus. Participants tapped on the location of the target using the touch screen to indicate when they detected a target. 
a) Assign 4 predictable target bins

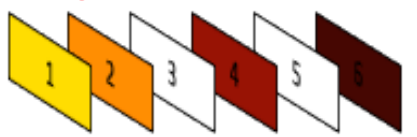

b)

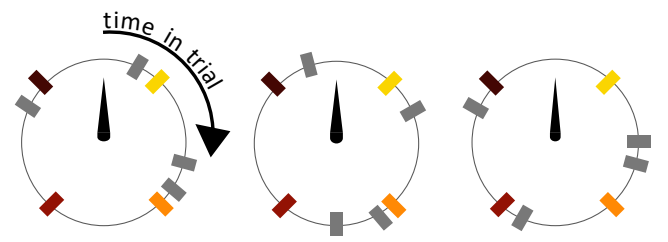

Eight targets on each trial

c)

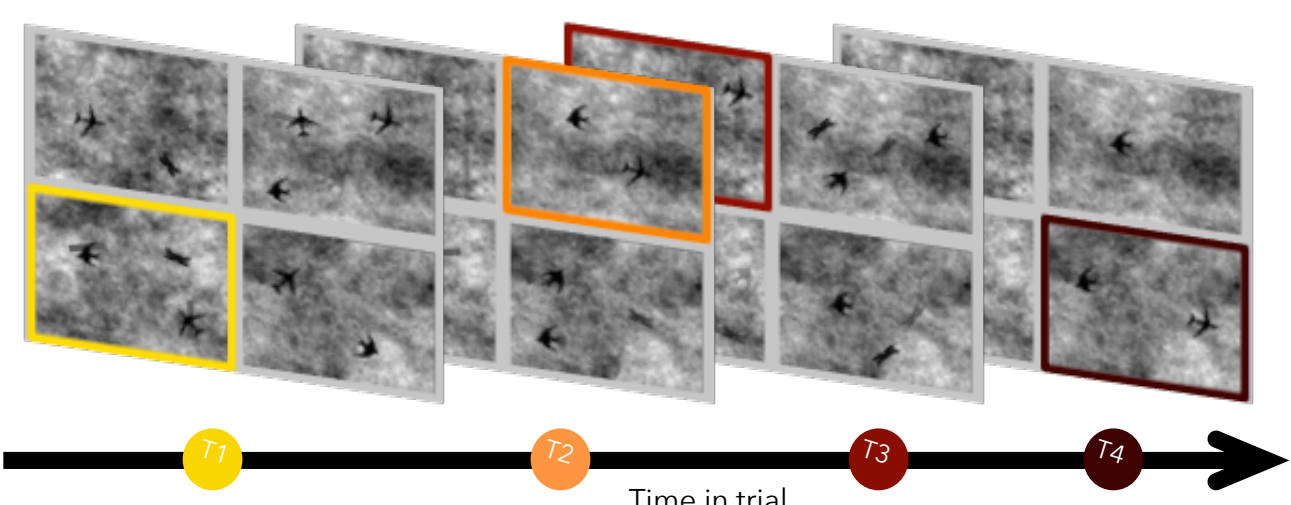

Time in trial

- 4 predictable targets appear in the same quadrant at the same time in each trial

- 4 unpredictable targets randomly distributed in time and space throughout each trial

Figure 1: The sequence of events within a trial. a) Each trial was divided into six time-bins. Four predictable targets appeared in each trial, pre-assigned in four of the six bins. b) The coloured dots represent predictable targets, which always occurred at the same time-point within their assigned bin across a block of trials. The grey dots represent the four random targets, which were randomly distributed over time. c) The four predictable targets were assigned to four different quadrants, which were kept constant throughout a block of trials. The quadrants in which random targets appeared were completely randomly determined.

Target predictability. Of the eight target stimuli, four were predictable. On every trial, they appeared with the same temporal onset from the start of the trial and within the same quadrant. Location within the quadrant, however, remained variable. The onset times for predictable targets were pre-assigned as follows: we created six time windows of $\sim 1$ second, with a gap of $\sim 660 \mathrm{~ms}$ between each neighbouring window (440 - 1440, 2100 - 3110, 3800 - 4800, 5470 - 6480, 7140-8140, 8810-9810 ms; times are relative to the onset of the trial, and rounded to the nearest ten milliseconds). Each window was then sub-divided into four equally spaced onsets. At the beginning of the experiment, each participant was allocated four different predictable onsets by first picking four bins out of six possible ones; and then choosing one onset (out of four) within each bin. Once these intervals and their quadrant association were selected, they remained fixed for that participant throughout the experiment. Different participants had different interval-quadrant pairings for predictable targets. The four unpredictable targets were distributed pseudorandomly, by assigning each to an onset derived from a uniform distribution 
across the full trial length. These constraints were set such that the targets were roughly evenly distributed throughout the trial, and to avoid too many target events occurring at one time.

Distractor predictability. In order to test for sensitivity to spatio-temporal regularities in unattended stimuli, half the distractors were also made predictable for one of the distractor categories (e.g., within the dragonfly but not bird category). Predictability for these distractors was assigned in the same way as for the target category, but independently (allowing some targets and some distractors to overlap in time, in some cases). Thus, there was no systematic relationship between target and distractor predictability.

Experimental procedure. Participants completed 10 practice trials to familiarise themselves with the game before completing a single block of 45 trials. Each trial contained 8 targets ( 4 predictable and 4 unpredictable). Overall, there were 360 targets (180 predictable and 180 unpredictable) per participant (excluding practice trials) and 720 distractors (180 predictable and 540 unpredictable). After each trial, observers received feedback in the form of a visual illustration: The screen depicted eight targets in a horizontal row that were coloured black for each target found and light grey for each target missed. These eight figures rotated, and a cheering sound was played when all the targets in a trial had been found. The feedback remained on the screen until tapping. When testing adults, they tapped to proceed at their own pace. When testing children, the experimenter tapped when the child was ready. The experimental block lasted approximately 15 minutes.

SWAN Questionnaire. Teachers completed the Strengths and Weaknesses of ADHDsymptoms and Normal-behaviours (SWAN) rating scale for each (Swanson et al., 2012). This is a well validated scale with 18 items measuring ADHD symptoms, with each item scored from -3 to +3 (below average to above average). Lower scores correspond to higher ADHD characteristics.

\section{Analyses}

Dependent variables: Our main dependant variable was hit rate. We focused on hit rate, rather than reaction times, because we did not instruct our participants to respond quickly, in order to avoid placing pressure on children and because hit rates proved a reliable and sensitive measure of benefits of spatiotemporal predictability 
in young adults in our previous study (Shalev, Boettcher, et al., 2019). In addition, to assess the effect of predictability on distractors, we also considered false alarm rates (i.e., tapping on distractors rather than targets).

Group Differences in Performance - Hit Rate: Hit rate was evaluated as a function of group (children vs. adults) and target predictability (predictable vs. unpredictable) to learn whether children and/or adults utilised regularities to improve performance, whether they differed in their overall performance, and whether there was an interaction between group and predictability. In addition, we modelled 'target order' to estimate behavioural effects over time on trial. This way, we could verify whether predictions influenced performance differentially for the first, second, third, or forth predictable vs. random targets.

Responses to target, classified as 'hit' or 'miss', were fitted using a generalized linear mixed-effects models (GLMMs) with a binomial distribution. Data were modelled using four fixed effects: Group (children vs. adults); Target Predictability (Predictable vs. Random targets); and Target Order (First/Second/Third/Forth position in the sequence, for each Predictable vs. Random targets). In the randomeffects structure, we included intercepts for each participant, as well as by-participant slopes for the effects of Target Predictability, Target Order, and their interaction.

Our model used the default 'logit' link function to map the relationship between the mean response and the linear combination of the predictors. The continuous Target Order factor was centred around zero by subtracting the grand average from each value (Aiken \& West, 1991; Enders \& Tofighi, 2007; Park, 2008). The GLMM was fitted with the maximum likelihood criterion, and we report the $t$ statistic for a hypothesis test contrasting each fixed-effects coefficient to a null hypothesis that the coefficient is equal to zero.

Group Differences in Performance - False Alarms: We applied a comparable analysis approach to false alarms, by quantifying the rate of tapping distractors. Here, we tagged each distractor as belonging to one of three categories, to distinguish distractors that appeared predictably ('Structured category - Predictable'), distractors that appeared randomly but were from the same category as the predictable distractors ('Structured category - Random'), and distractors that appeared randomly and belonged to a different category ('Unstructured category - Random'). We used GLMM, with the Distractor Category and Group (children vs. adults) as the fixedeffects. In the random-effects structure we included intercepts for each participant, 
as well as by-participant slopes for the effect of Distractor Category and their interaction.

Individual differences: We applied a series of analyses to identify whether our dynamic visual search task was sensitive to individual differences within the group of children. In these analyses, we focused on the hit-rate variable, which can reveal an interesting dissociation between 'predictions' and overall hit rate. Two continuous variables were used to mark individual differences: chronological age (in months) and the combined SWAN score. These variables were added to a GLMM as fixed-effects covariates, alongside Target Predictability. The random-effects structure included intercepts for each participant, as well as by-participant slopes for Target Predictability and their interaction. As in the previous section, the model used the default 'logit' link function. The continuous variables (Age and SWAN score) were centred around zero by subtracting the grand average from each value (Aiken \& West, 1991; Enders \& Tofighi, 2007; Park, 2008). The GLMM was fitted with the maximum likelihood criterion, and we report the t-statistic for a hypothesis test contrasting each fixed-effects coefficient to a null hypothesis that the coefficient is equal to zero.

\section{Data exclusion}

Two children did not complete the experiment and were excluded. Altogether, our final sample included 78 children and 25 adults.

\section{Results}

\section{Contrasting group performance}

Our first analysis focused on the contribution of target predictability and serial position to hit rate, according to group. The results are illustrated in Figure 2. The GLMM indicated a main effect of Target Predictability $(t(37072)=3.99 ; p<.001$; $95 \% \mathrm{Cl}[.05 ; .16])$, a main effect of Group (t(37072)=16.33; $p<.001 ; 95 \% \mathrm{Cl}[.75 ; .96])$, and a main effect of Target $\operatorname{Order}(\mathrm{t}(37072)=-4.80 ; p<.001 ; 95 \% \mathrm{Cl}[-.16 ;-.06])$. There were no interactions between factors. Group did not interact with Target Predictability $(p=.38)$ or with Target Order $(p=.23)$. Target Order did not interact with Target Predictability $(p=.65)$, and there was no three-way interaction among the factors $(p=.87)$. Altogether, these results reflect a higher hit rate when targets appeared predictably, a higher hit rate in the group of adults, and differences in the mean hit rate at different time points along the trial. Critically, there were no interactions, 
meaning there were no evidence that either the group or the ordinal position of the target influenced performance benefits.
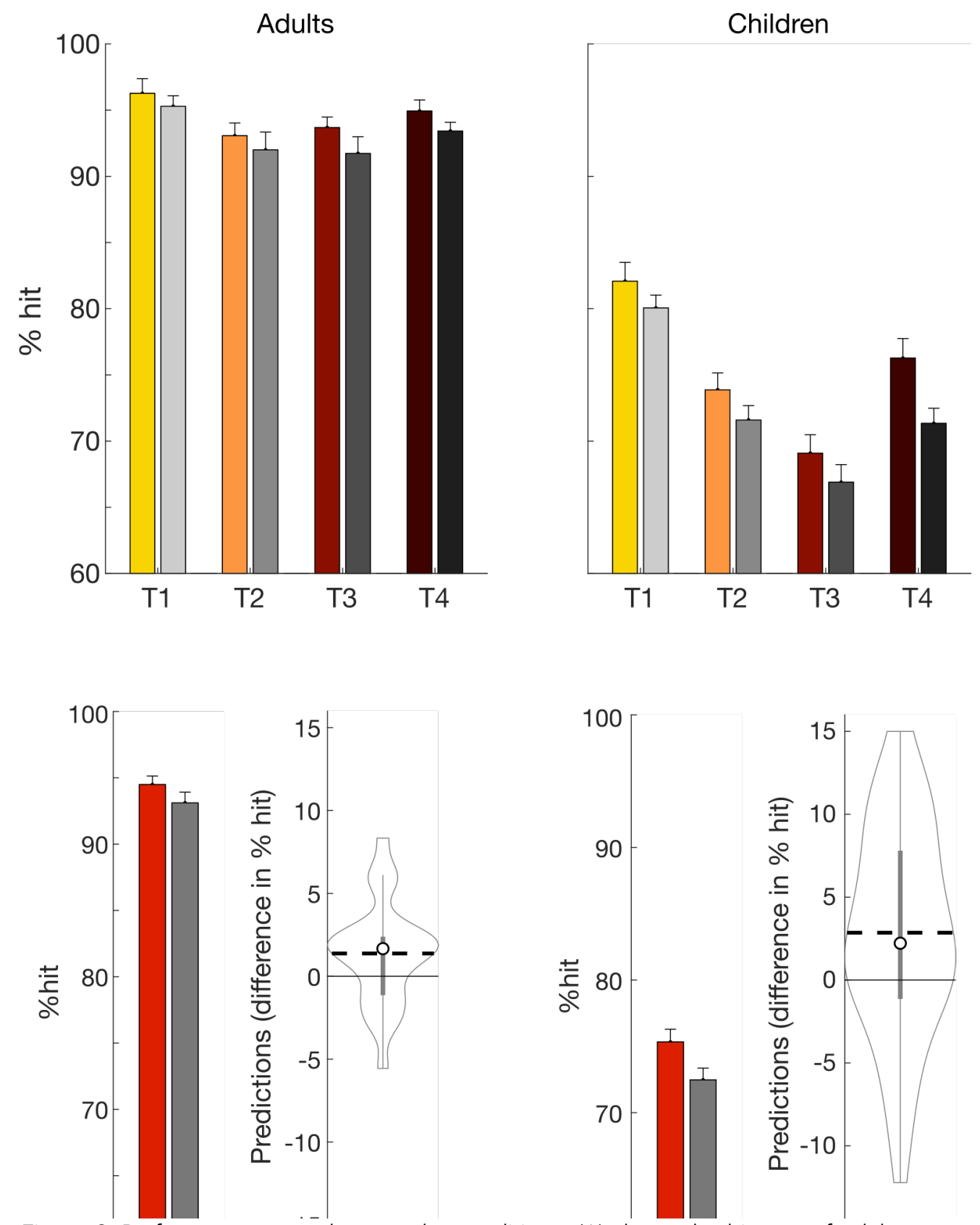

Figure 2: Performance on each group by conditions. (A) shows the hit rate of adults on predictable targets (coloured bars) vs. unpredictable (grey bars), splitting the trial into four equal time-windows, to observe the benefit of predictions at different time-points along each trial. Error-bars, in all bar plots, represent standard error of the mean; (B) shows the same contrast among children; (C) overall hit-rate for predictable (red) vs. unpredictable targets (grey); the data in (D) depicts the difference between hitting predictable and unpredictable targets at the individual level, using a violin plot. The width represent the number of observations; The circle represents the group mean; horizontal dashed line represents the group median; bold vertical line is the interquartile range, and the thinner vertical line represents the range between higher and lower adjacent values; figures $(E)$ and $(F)$ depict the same as $(C)$ and $(D)$ among the group of children. 
The next analysis used comparable modelling to contrast the rate of false alarms in the two groups by Distractor Category (see Figure 3). The GLMM results for the rate of false alarms indicated a main effect of Group ( $t(74152)=3.64 ; \quad p<.001$; $95 \% \mathrm{Cl}[.51 ; 1.71])$, reflecting a significantly higher rate of false alarms for children. There was no main effect of Distractor Type $(p=.85)$ and no Group X Distractor Type interaction $(p=.89)$.
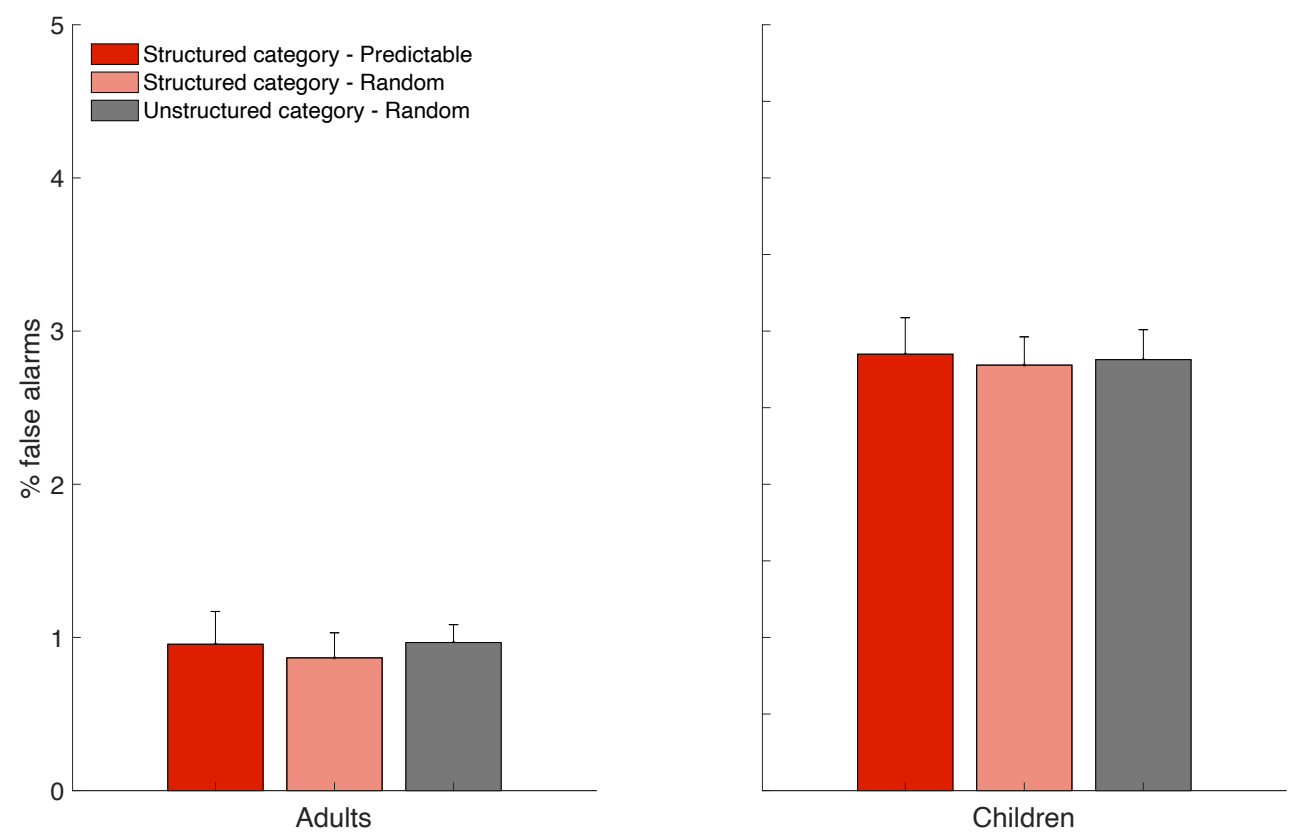

Figure 3: false alarm errors (selecting distractors instead of targets), split by three experimental conditions in two groups. Distractors that were belong to the "Structured category - Predictable" (red) are four distractors that appeared regularly, similar to the predictable targets; The "Structured category - Random" (light coral) were four distractors of the same category as the predictable ones (either aeroplanes, birds, or bugs) however they were randomly distributed in time and space; the "Unstructured category - Random" were the other eight targets, of a different category, and were all distributed randomly. Error bars represent standard error of the mean.

\section{Individual differences among children}

We tested for individual differences in overall hit rate and benefits of predictions in the group of children. The data are presented in figures $4 a-d$, in which scatter plots depict the relationships between the SWAN and Age factors as predictors of overall hit rate and of 'prediction benefit' (defined as the difference between hit rate for predictable and unpredictable targets).

The statistical analyses tested for significant linear relationships between Target Predictability (Predictable vs. Random), age (in months), teachers' scores on the SWAN questionnaire, and the hit rate as the dependant variable, using a single 
GLMM. The results replicated the main effect of Target Predictability when focusing exclusively on the group of children $(t(27712)=4.09 ; p<.001 ; 95 \% \mathrm{CI}[.04 ; .11])$. There was also a main effect of the Age (in months) covariate $(t(27712)=2.26 ; p=.02$; $95 \% \mathrm{Cl}[.00 ; .04])$, with a positive coefficient $(.025 ; \mathrm{SE}=.01)$ indicating a positive relationship between advancing age and overall hit rate. Whereas there was no main effect of SWAN score $(p=.56)$, there was a significant interaction between the SWAN and Target Predictability $(t(27712)=2.59 ; p=.009 ; 95 \% \mathrm{Cl}[.000 ; .005])$. Age did not interact with Target Predictability $(p=.75)$, and there were no other significant interactions (all $p^{\prime} s$ >.1). To interpret the interaction between SWAN and Target Predictability, we calculated a composite score for Prediction Benefit by subtracting the hit rate for random targets from predictable ones. We then carried a confirmatory analysis to estimate the linear correlation between the two variables, which was positive and significant $(r(77)=.23 ; p=.047)$, indicating a greater benefit of predictability for individuals with higher SWAN score (lower ADHD symptoms). Importantly, Age and SWAN score did not correlate $(p=.25)$. 

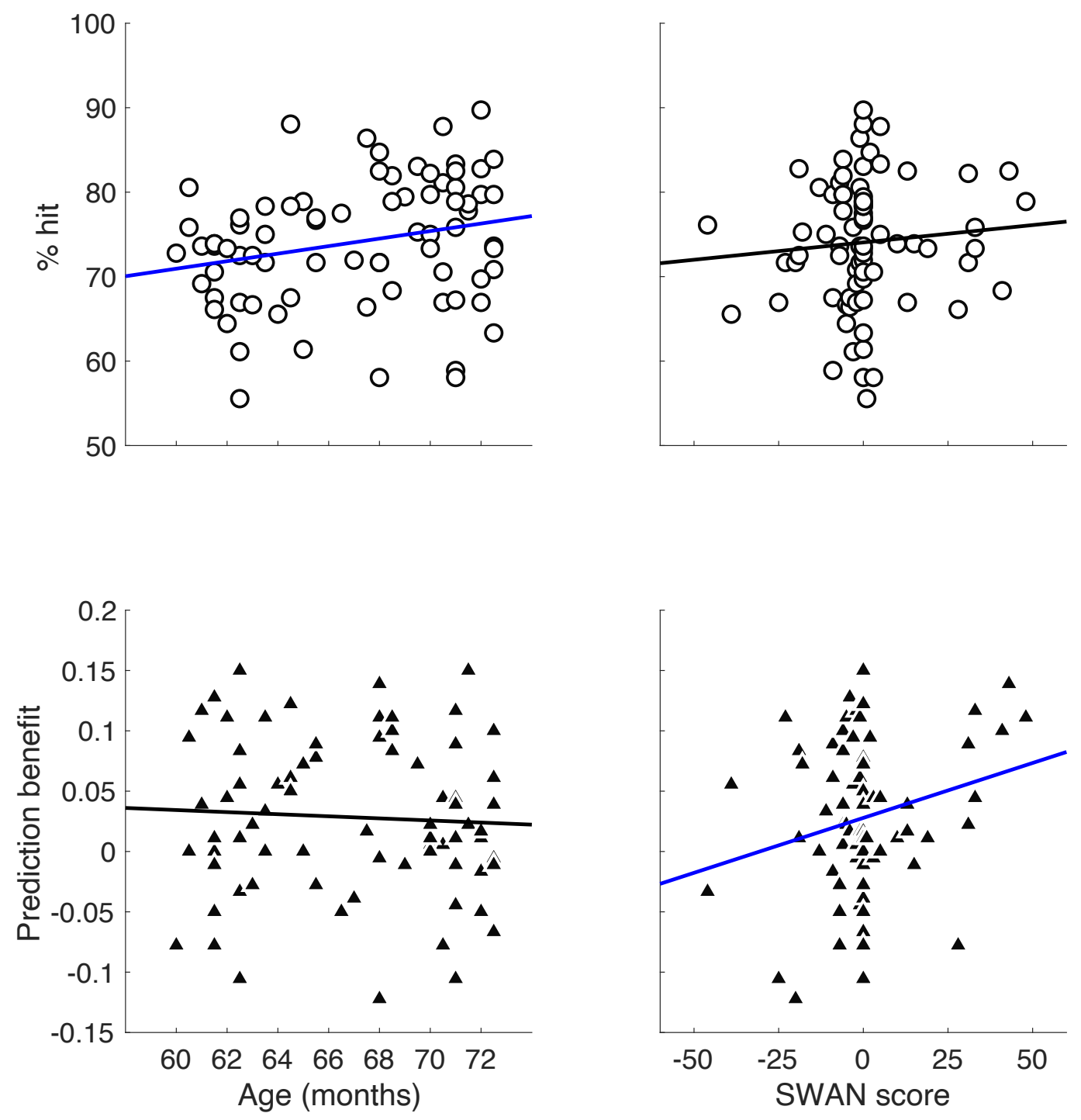

Figure 4: scatter plots testing the association between performance measures, age, and SWAN score. Top row shows relationships involving overall mean hit rate. Overall hit rates correlated significantly with age but not SWAN score. Bottom row shows relationships involving prediction benefit. Prediction benefit correlated/covaried significantly with SWAN (.047) score but not age (.25).

\section{Discussion}

Although young children showed lower overall ability of detecting targets and ignoring distractors compared to adults in our extended dynamic visual search task, they nevertheless benefited from spatiotemporal regularities as much as adults. Our results suggest a more nuanced interpretation of the relationship between learning and top-down control than has previously been suggested. In the context of our task, the learning and utilisation of regularities were selective and used adaptively to guide 
goal-driven behaviour just as well in children as in adults. Children, like adults, did not show any bias towards task-irrelevant regularities, while at the same time they learned relevant regularities that served anticipation-based guidance.

Differences in spatial attention were reflected in the overall lower hit rate and higher false-alarm rate in children when compared to adults. We also identified a significant correlation between hit rate and age within the group of children. This provides further, more fine-grained, evidence of how spatial search develops during childhood. This pattern may suggest particular difficulty with competition and distraction among children, in line with existing literature (Kim \& Kastner, 2019). We observed that lowest performance levels occurred in the middle of trial (reflected in the main effect of Target Order), when overall the likelihood of distractors peaked. As a tentative explanation, this may result from the maximum potential for overlap among stimuli at different stages of appearance is in the middle due to the dynamic nature of the task, in which stimuli appear and disappear over extended time. However, it is of note that, even during this period, both children and adults exhibited a benefit for predictable compared to unpredictable targets. In the current experiment, we did not manipulate the parameter of number of co-occurring distractors systematically. However, the experimental approach we used is suitable for designs that specifically control the degree of competition across or within trials, which should prove informative in future studies.

In contrast to age differences in overall hit rate in visual search, children showed reliable and persistent benefits of spatiotemporal expectation, which did not differ in magnitude from those we found in young adults. This pattern extends previous reports on the ability of children to learn patterns and utilise predictions. Previous work focused on discrete stimulus-interval associations in cueing tasks as a source of anticipation (e.g., Droit-Volet \& Coull, 2016; Johnson, Burrowes, \& Coull, 2015; Mento \& Granziol, 2020; Vallesi \& Shallice, 2007). In the statistical learning literature, learning of visual regularities is normally confined to single objects and shape co-occurrence, while temporal intervals are kept constant (e.g., Fiser \& Aslin, 2002; Kirkham, Slemmer, \& Johnson, 2002; Wu, Gopnik, Richardson, \& Kirkham, 2011). Here we demonstrate that children can learn patterns of task-relevant spatiotemporal information presented among dynamic distractors, and utilise anticipation to benefit performance in that context.

Another important addition we make to the current literature on anticipationled behaviour in children pertains to the nature of structures that are learned and utilised. In our dynamic task, the behavioural benefit resulted from encoding temporal intervals (in contrast to serial order, as often used in studies of statistical learning). 
Furthermore, the regular temporal intervals were embedded within, and had to be extracted from, the context of many intervening unpredictable stimuli occurring across time and space. Learning single simple temporal associations would therefore be insufficient to confer any behavioural advantage. Instead, to benefit performance, participants had to abstract the timing and location of predictable relevant events from the unfolding succession of unpredictable targets and distractors. In addition, orienting of attention combined the temporal and spatial dimensions, in contrast to most previous studies on temporal attention in which target location is equated across trials - i.e. the discrete events occurring at a consistent location.

Interestingly, even though children were overall worse than adults, task irrelevant, yet predictable stimuli did not yield more erroneous responses compared to their unpredictable counterparts. This is in contrast to previous reports of automatic capture by regularities (Zhao, Al-Aidroos, \& Turk-Browne, 2013). In our task such an effect would have been manifested by a larger false-alarm rate for predictable distractors. If children were particularly susceptible to predictions, their capture by predictable distractors might be even stronger. This was not observed. We cannot completely rule out the possibility that our pattern of predictability was either too subtle or too complex to induce capture by task-irrelevant information. However, overall our findings accord with studies in adults showing that effects of predictions are strongly modulated by task goals (Boettcher, Stokes, Nobre, \& van Ede, 2020; Shalev, Nobre, \& van Ede, 2019; Stokes, Myers, Turnbull, \& Nobre, 2014). What is somewhat surprising is that we observed these patterns in young children. In our task, instead of sensitivity to patterns working against focused, goal-oriented behaviour (Gaspelin et al., 2015), expectations only benefitted performance.

The benefit of expectations was tested within the group of children, in whom we modelled the data with two potential covariates: age and SWAN questionnaire (an ADHD symptom scale, used as a proxy of typical age-appropriate attention development). The model results revealed a dissociation between task markers related to age (overall hit rate) vs. with typical attention development (prediction benefits). Our findings suggested the ability to form predictions does not change much with age (at least within the range between 5 to 6 years of age), but instead follows a proxy for age-appropriate manifestation of attention. While in the current study we did not target behavioural syndromes as ADHD, the data is in line with a recent report showing a selective deficit in temporal preparation among adults diagnosed with ADHD (e.g., Dankner, Shalev, Carrasco, \& Yuval-Greenberg, 2017; Hasler et al., 2016; Johnson et al., 2008; McAvinue et al., 2015; Vallesi et al., 2016). However, direct implications of our data for an understanding of ADHD should be 
treated with caution. Our sample included relatively young children who had no reports of any clinical diagnosis, and therefore this point can be addressed further in future studies with more diverse samples.

In conclusion, here we tested for the first time how children guide spatial attention in a dynamic, continuous context, based on complex task-embedded regularities amidst distraction. Whereas in line with previous studies children were less efficient in finding targets when compared to adults, they used predictability to guide attention very effectively. This effect was robust and consistent throughout extended trials, revealing a marked capacity of encoding complex temporal structures, and sustaining goal-driven guidance of selective attention in time. Moreover, we did not find capture by regularities embedded in task-irrelevant information (predictable distractors), providing further evidence to support highly controlled behaviour by young children. These robust effects were observed using a short, playful task, which can be a useful method for future explorations. One fruitful avenue could be investigating individual differences, which we managed to reveal within a single cohort of neurotypically developing 5 to 6 year-olds. We believe that our design provides a step forward towards understanding selective attention in ecological settings, in which the temporal dimension is inseparable from spatially oriented behaviour.

\section{References}

Aiken, L. S., \& West, S. G. (1991). The effects of predictor scaling on coefficients of regression equations. Multiple Regression: Testing and Interpreting Interactions.

Amso, D., \& Scerif, G. (2015). The attentive brain: Insights from developmental cognitive neuroscience. Nature Reviews Neuroscience. https://doi.org/10.1038/nrn4025

Bertels, J., San Anton, E., Gebuis, T., \& Destrebecqz, A. (2017). Learning the association between a context and a target location in infancy. Developmental Science. https://doi.org/10.1111/desc.12397

Boettcher, S. E. P., Stokes, M. G., Nobre, A. C., \& van Ede, F. (2020). One thing leads to another: Anticipating visual object identity based on associativememory. Journal of Neuroscience. https://doi.org/10.1523/JNEUROSCI.275119.2020

Brackbill, Y., \& Fitzgerald, H. E. (1972). Stereotype Temporal Conditioning in Infants. Psychophysiology. https://doi.org/10.1111/j.1469-8986.1972.tb00766.x

Brainard, D. H. (1997). The Psychophysics Toolbox. Spatial Vision, 10(4), 433-436. Colombo, J. (2001). The Development of Visual Attention in Infancy. Annual Review of Psychology, 52(1), 337-367. https://doi.org/10.1146/annurev.psych.52.1.337 
Colombo, J., \& Richman, W. A. (2002). Infant timekeeping: Attention and temporal estimation in 4-month-olds. Psychological Science.

https://doi.org/10.1111/1467-9280.00484

Dankner, Y., Shalev, L., Carrasco, M., \& Yuval-Greenberg, S. (2017). Prestimulus Inhibition of Saccades in Adults With and Without Attention-

Deficit/Hyperactivity Disorder as an Index of Temporal Expectations.

Psychological Science, 28(7), 835-850.

https://doi.org/10.1177/0956797617694863

Donnelly, N., Cave, K., Greenway, R., Hadwin, J. A., Stevenson, J., \& Sonuga-Barke,

E. (2007). Visual search in children and adults: Top-down and bottom-up mechanisms. Quarterly Journal of Experimental Psychology.

https://doi.org/10.1080/17470210600625362

Droit-Volet, S., \& Coull, J. T. (2016). Distinct developmental trajectories for explicit and implicit timing. Journal of Experimental Child Psychology.

https://doi.org/10.1016/j.jecp.2016.05.010

Enders, C. K., \& Tofighi, D. (2007). Centering Predictor Variables in Cross-Sectional Multilevel Models: A New Look at an Old Issue. Psychological Methods. https://doi.org/10.1037/1082-989X.12.2.121

Estes, K. G., Evans, J. L., Alibali, M. W., \& Saffran, J. R. (2007). Can infants map meaning to newly segmented words? Statistical segmentation and word learning. Psychological Science. https://doi.org/10.1111/j.14679280.2007.01885.x

Fiser, J., \& Aslin, R. N. (2002). Statistical learning of new visual feature combinations by infants. Proceedings of the National Academy of Sciences of the United States of America. https://doi.org/10.1073/pnas.232472899

Gaspelin, N., Margett-Jordan, T., \& Ruthruff, E. (2015). Susceptible to distraction: Children lack top-down control over spatial attention capture. Psychonomic Bulletin and Review. https://doi.org/10.3758/s13423-014-0708-0

Gopnik, A., O'Grady, S., Lucas, C. G., Griffiths, T. L., Wente, A., Bridgers, S., ... Dahl, R. E. (2017). Changes in cognitive flexibility and hypothesis search across human life history from childhood to adolescence to adulthood. Proceedings of the National Academy of Sciences of the United States of America. https://doi.org/10.1073/pnas.1700811114

Hasler, R., Perroud, N., Meziane, H. B., Herrmann, F., Prada, P., Giannakopoulos, P., \& Deiber, M. P. (2016). Attention-related EEG markers in adult ADHD.

Neuropsychologia. https://doi.org/10.1016/j.neuropsychologia.2016.05.008 Hommel, B., Li, K. Z. H., \& Li, S. C. (2004). Visual search across the life span. Developmental Psychology. https://doi.org/10.1037/0012-1649.40.4.545 Johnson, K. A., Burrowes, E., \& Coull, J. T. (2015). Children can implicitly, but not voluntarily, direct attention in time. PLOS ONE. https://doi.org/10.1371/journal.pone.0123625

Johnson, K. A., Robertson, I. H., Barry, E., Mulligan, A., Dáibhis, A., Daly, M., ... 
Bellgrove, M. A. (2008). Impaired conflict resolution and alerting in children with ADHD: evidence from the Attention Network Task (ANT). Journal of Child Psychology and Psychiatry, and Allied Disciplines. https://doi.org/10.1111/j.1469-7610.2008.01936.x

Johnson, M. H., Posner, M. I., \& Rothbart, M. K. (1991). Components of visual orienting in early infancy: Contingency learning, anticipatory looking, and disengaging. Journal of Cognitive Neuroscience. https://doi.org/10.1162/jocn.1991.3.4.335

Johnson, Mark H. (2001). Functional brain development in humans. Nature Reviews Neuroscience. https://doi.org/10.1038/35081509

Kim, N. Y., \& Kastner, S. (2019). A biased competition theory for the developmental cognitive neuroscience of visuo-spatial attention. Current Opinion in Psychology. https://doi.org/10.1016/j.copsyc.2019.03.017

Kirkham, N. Z., Slemmer, J. A., \& Johnson, S. P. (2002). Visual statistical learning in infancy: Evidence for a domain general learning mechanism. Cognition. https://doi.org/10.1016/S0010-0277(02)00004-5

Lobaugh, N. J., Cole, S., \& Rovet, J. F. (1998). Visual search for features and conjunctions in development. Canadian Journal of Experimental Psychology. https://doi.org/10.1037/h0087293

Lucas, C. G., Bridgers, S., Griffiths, T. L., \& Gopnik, A. (2014). When children are better (or at least more open-minded) learners than adults: Developmental differences in learning the forms of causal relationships. Cognition. https://doi.org/10.1016/j.cognition.2013.12.010

McAvinue, L. P., Vangkilde, S., Johnson, K. A., Habekost, T., Kyllingsbæk, S., Bundesen, C., \& Robertson, I. H. (2015). A Componential Analysis of Visual Attention in Children With ADHD. Journal of Attention Disorders. https://doi.org/10.1177/1087054712461935

Mento, G., \& Granziol, U. (2020). The developing predictive brain: How implicit temporal expectancy induced by local and global prediction shapes action preparation across development. Developmental Science. https://doi.org/10.1111/desc.12954

Mento, G., \& Valenza, E. (2016). Spatiotemporal neurodynamics of automatic temporal expectancy in 9-month old infants. Scientific Reports. https://doi.org/10.1038/srep36525

Merrill, E. C., \& Lookadoo, R. (2004). Selective search for conjunctively defined targets by children and young adults. Journal of Experimental Child Psychology. https://doi.org/10.1016/j.jecp.2004.04.001

Ólafsdóttir, I. M., Kristjánsson, T., Gestsdóttir, S., Jóhannesson, Ó. I., \& Kristjánsson, Á. (2016). Understanding visual attention in childhood: Insights from a new visual foraging task. Cognitive Research: Principles and Implications. https://doi.org/10.1186/s41235-016-0016-5

Park, H. S. (2008). Centering in Hierarchical Linear Modeling. Communication 
Methods and Measures. https://doi.org/10.1080/19312450802310466

Plebanek, D. J., \& Sloutsky, V. M. (2017). Costs of Selective Attention: When

Children Notice What Adults Miss. Psychological Science.

https://doi.org/10.1177/0956797617693005

Saffran, J. R. (2001). Words in a sea of sounds: The output of infant statistical

learning. Cognition. https://doi.org/10.1016/S0010-0277(01)00132-9

Saffran, J. R., \& Kirkham, N. Z. (2018). Infant Statistical Learning. Annual Review of

Psychology. https://doi.org/10.1146/annurev-psych-122216-011805

Scerif, G. (2010). Attention trajectories, mechanisms and outcomes: at the interface between developing cognition and environment. Developmental Science, 13(6), 805-812.

Shalev, N., Boettcher, S. E. P., \& Nobre, A. C. (2019). Right time, right place: implicit learning of target onsets in a visual search task. Journal of Vision. https://doi.org/10.1167/19.10.255b

Shalev, N., Nobre, A. C., \& van Ede, F. (2019). Time for What? Breaking Down Temporal Anticipation. Trends in Neurosciences. https://doi.org/10.1016/j.tins.2019.03.002

Sloutsky, V. M., \& Fisher, A. V. (2004). When development and learning decrease memory: Evidence against category-based induction in children. Psychological Science. https://doi.org/10.1111/j.0956-7976.2004.00718.x

Stokes, M. G., Myers, N. E., Turnbull, J., \& Nobre, A. C. (2014). Preferential encoding of behaviorally relevant predictions revealed by EEG. Frontiers in Human Neuroscience. https://doi.org/10.3389/fnhum.2014.00687

Swanson, J. M., Schuck, S., Porter, M. M., Carlson, C., Hartman, C. A., Sergeant, J. A., ... Wigal, T. (2012). Categorical and Dimensional Definitions and Evaluations of Symptoms of ADHD: History of the SNAP and the SWAN Rating Scales. The International Journal of Educational and Psychological Assessment.

Thiessen, E. D. (2017). What's statistical about learning? Insights from modelling statistical learning as a set of memory processes. Philosophical Transactions of the Royal Society B: Biological Sciences. https://doi.org/10.1098/rstb.2016.0056

Trick, L. M., \& Enns, J. T. (1998). Lifespan changes in attention: The visual search task. Cognitive Development. https://doi.org/10.1016/S0885-2014(98)90016-8

Tummeltshammer, K., Amso, D., French, R. M., \& Kirkham, N. Z. (2017). Across space and time: infants learn from backward and forward visual statistics.

Developmental Science. https://doi.org/10.1111/desc.12474

Vallesi, A., D'Agati, E., Grelloni, C., Pasini, A., Mazzotta, G., \& Curatolo, P. (2016).

Effect of modified-release methylphenidate on cognition in children with ADHD: Evidence from a temporal preparation task. Timing and Time Perception. https://doi.org/10.1163/22134468-00002067

Vallesi, A., \& Shallice, T. (2007). Developmental Dissociations of Preparation Over Time: Deconstructing the Variable Foreperiod Phenomena. Journal of 
Experimental Psychology: Human Perception and Performance. https://doi.org/10.1037/0096-1523.33.6.1377

Whitebread, D., \& Neilson, K. (2000). The contribution of visual search strategies to the development of pedestrian skills by 4-11 year-old children. British Journal of Educational Psychology. https://doi.org/10.1348/000709900158290

Wolfe, J. M. (2020). Visual Search: How Do We Find What We Are Looking For? Annual Review of Vision Science. https://doi.org/10.1146/annurev-vision091718-015048

Wu, R., Gopnik, A., Richardson, D. C., \& Kirkham, N. Z. (2011). Infants Learn About Objects From Statistics and People. Developmental Psychology. https://doi.org/10.1037/a0024023

Zhao, J., Al-Aidroos, N., \& Turk-Browne, N. B. (2013). Attention Is Spontaneously Biased Toward Regularities. Psychological Science, 24(5), 667-677. https://doi.org/10.1177/0956797612460407 